Session 4: From the AGB to the WD stellar phases Chair: Miriam Pena 


\title{
Progress and Application of The Initial-Final Mass Relation
}

\author{
Jeffrey D. Cummings \\ Center for Astrophysical Sciences, Johns Hopkins University \\ 3400 N Charles Street \\ Baltimore, MD 21218, USA \\ email: jcummi19@jhu.edu
}

\begin{abstract}
The stellar life cycle is dominated by phases such as the hydrogen-burning stage and the remnant white dwarf cooling phase. However, between these two stages, stars dramatically transform themselves by losing the bulk of their mass. Planetary nebulae (PNe) provide a powerful clue to the processes involved in this transformation, but they are very complex. Over the past 15 years, a new wave of imaging and spectroscopy programs have uncovered the remnants of $\mathrm{PNe}$, white dwarfs, in a wide range of well-measured environments. With this we can map the masses and temperatures of the stellar remnants to the properties of their progenitors. This work has now led to the first uniform mapping of the initial-final mass relation from 1.5 to $7 \mathrm{M}_{\odot}$. The resulting relation is a fundamental input to our understanding of stellar evolution for low and intermediate-mass stars that produce PNe and has a wide range of applications.
\end{abstract}

Keywords. White Dwarfs, AGB and post-AGB, Planetary Nebula, Mass Loss

\section{Introduction}

White dwarfs provide tools to help understand the late stages of stellar evolution. They help most critically with the challenging asymptotic giant branch (AGB) stars and planetary nebulae $(\mathrm{PNe})$ phase. AGB stars and PNe are very bright but extremely brief stages in stellar evolution ( $\sim$ a few million years or less and tens of thousand years, respectively), and their complexity makes both modeling them and observationally disentangling their characteristics challenging. White dwarfs, while significantly fainter, are extremely long lived and relatively numerous. Additionally, we can directly observe them without having to consider significant effects of the surrounding nebulosity from mass loss.

Deriving the intrinsic properties of white dwarfs can be done with low resolution spectra of their Balmer lines or He features. A majority of white dwarfs have pure Hydrogen (DA) but an important fraction have pure Helium (DB) atmospheres. This results from the rapid gravitational settling of all heavier elements. This gives white dwarfs simple spectra with multiple strong and broad Balmer features (e.g., Bergeron et al. 1992) or Helium features (e.g., Beauchamp et al. 1996) in the blue (3700 to $5200 \AA$ ). These features are pressure sensitive and fitting them all simultaneously can precisely derive both the $\mathrm{T}_{\text {eff }}$ and surface gravities of these very compact and typically very hot objects. This technique has become far more common than other methods to estimate parameters, those being estimates from multi-color photometry (e.g., Wegner 1983), gravitational redshift (e.g., Wegner 1989), and white dwarfs in binaries. These methods, however, still provide important consistency checks and in certain contexts can be very useful.

Direct application of the $\mathrm{T}_{\text {eff }}$ and surface gravities to white dwarf cooling models (e.g., Fontaine et al. 2001) derives the mass, cooling age, luminosity, and color of the white dwarf. The cooling models are relatively simple, but they have important sensitivity to the white dwarf compositions. For example, the most massive white dwarfs are believed 
to have oxygen-neon (ONe) cores (Althaus et al. 2007) instead of the more common carbon-oxygen $(\mathrm{CO})$ cores formed from lower-mass stars that cannot ignite off-center carbon burning in their cores (Garcia-Berro et al. 1997). ONe core white dwarfs cool more rapidly and have higher surface gravities than $\mathrm{CO}$ white dwarfs of the same mass would. The white dwarf mass at which this transition occurs remains uncertain, but it is approximately $1.1 \mathrm{M}_{\odot}$.

Being able to measure spectroscopically both the intrinsic white dwarf masses and cooling ages provides many tools to studying the historical characteristics and distributions of stellar populations (e.g., Tremblay et al. 2014; 2016), and to infer information about each white dwarf's progenitor. One of the most critical relations for understanding stellar evolution is the initial-final mass relation (IFMR), which is a direct comparison of the mass that a star forms with on the main sequence to its final mass that it will have as a white dwarf. The IFMR provides a direct measurement of the total mass loss during a star's life and how it varies with initial mass, and with more detailed analysis, how it varies with metallicity. This gives critical constraints to AGB stars (e.g., Kalirai et al. 2014), PNe (e.g., Ciardullo 2010), and to our understanding of supernovae and their rates (e.g., Greggio et al. 2010). The IFMR, however, remained poorly constrained for many years. After significant work on the relation in the past 15 years its applications are now more reliable, but it still has several important limitations.

\section{The Initial-Final Mass Relation}

\subsection{A Brief History}

One of the first detailed looks at the IFMR was the analysis of Weidemann (1977). Weidemann merged the results of a small number of studied white dwarfs at the time. These being LB 1497 from the Pleiades (Schulz 1976), seven white dwarfs from the Hyades (Eggen \& Greenstein 1965; van Altena 1969; Sweeney 1976), and three well studied white dwarfs in binaries. Weidemann found a generally consistent trend with increasing $\mathrm{M}_{\text {initial }}$ creating more massive white dwarfs. Weidemann's primary goal in this analysis was to compare this semi-empirical relation to the evolutionary and mass-loss models of the time (e.g., Paczyński 1970; Fusi-Pecci \& Renzini 1976; Wood \& Cahn 1977). It was consistently found that to recreate the IFMR of Weidemann (1977) required higher mass loss than nearly all models of the time predicted. However, the relation was still limited by very small numbers and limitations in the techniques used to derive both initial and final masses.

In the 80 s and 90 s progress with the IFMR was steady but slow due to the challenges of finding new cluster white dwarfs and getting deep spectroscopy for precision analysis. Focus was put on the Hyades (e.g., Reid 1996), NGC 3532 (e.g., Reimers \& Koester 1989; Koester \& Reimers 1993), and NGC 2516 (Koester \& Reimers 1996). With significantly improved models and techniques to determine white dwarf parameters (Bergeron et al. 1992), the masses and cooling ages were meaningfully adjusted. For example, spectroscopic redeterminations of the mass of the Pleiades's LB 1497 increased its previously measured $0.9 \mathrm{M}_{\odot}$ to $1.08 \mathrm{M}_{\odot}$ (Bergeron et al. 1995). With these data Weidemann et al. (2000) found a more precise but still sparse and limited IFMR.

With the advent of wide-field imagers and multi-object spectrographs on larger telescopes over the past 15 years, there has been an explosion of new white dwarf data. Namely in NGC 2099 (Kalirai et al. 2005), Praesepe (Dobbie et al. 2006a; Casewell et al. 2009), NGC 6791 (Kalirai et al. 2007), NGC 6819 and NGC 7789 (Kalirai et al. 2008), NGC 2287 and NGC 3532 (Dobbie et al. 2009), NGC 2618 (Williams et al. 2004; 2009), 


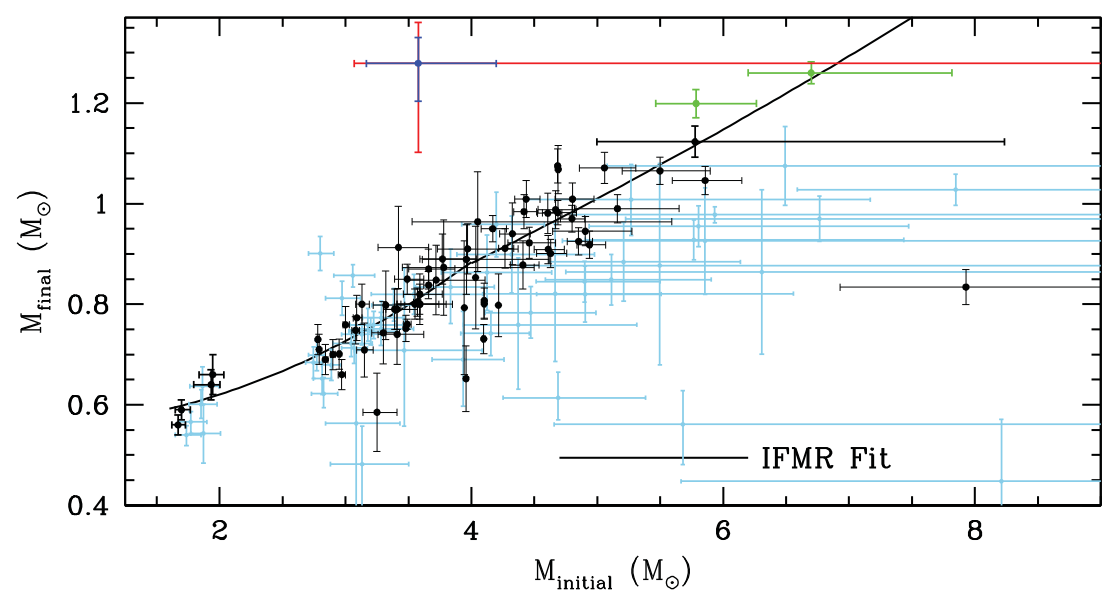

Figure 1. The IFMR combining the work of Kalirai et al. (2014) and Cummings et al. (2015; 2016a; 2016b), which analyzed newly discovered white dwarfs and uniformly analyzed previous data publicly available. Green points show the Pleiades white dwarfs connected only kinematically to the cluster (Dobbie et al. 2006b). The blue point shows the ultramassive NGC 2099 white dwarf that deviates significantly, likely due to its old age introducing significant errors (blue $1 \sigma$, red $2 \sigma$ ). Comparison to the IFMR of Salaris et al. (2009; light blue) shows that newly discovered white dwarfs and precision analysis of the photometry and spectroscopy increases the sample by $\sim 50 \%$ and reduces the scatter by $\sim 50 \%$. (See online version for Figure 1 in color.)

and the globular cluster NGC 6121 (Kalirai et al. 2009). This more than doubled the number of analyzed cluster white dwarfs in the IFMR from 2000 to 2009.

\subsection{Recent Progress}

Salaris et al. (2009), Catalán et al. (2008), and Kalirai et al. (2009) each looked at the now large samples of known white dwarfs in open clusters, combining the work of all previous studies. There remained two critical limitations in these semi-empirical relations. First, there were two large gaps in the relation at $\mathrm{M}_{\text {initial }}$ of 2 to $2.75 \mathrm{M}_{\odot}$ and for masses greater than 6 to $7 \mathrm{M}_{\odot}$ (e.g., $\left.\mathrm{M}_{\mathrm{final}}>1.05 \mathrm{M}_{\odot}\right)$. This intermediate mass range requires observations of white dwarfs in star clusters of $\sim 1$ Gyr, where stars of these masses have had enough time to fully evolve and the resulting white dwarfs have not significantly cooled. For the highest-mass gap, the question remained, where are the high-mass white dwarfs? The second limitation was that the scatter in the relations were large $\left( \pm 0.10 \mathrm{M}_{\odot}\right)$. Because these white dwarfs come from multiple clusters and studies from multiple independent researchers, is this scatter simply the result of systematic differences between the analyses? Salaris et al. (2009) considered in more depth the adopted ages of the host clusters, but the scatter remained. Or is this persistent scatter indicative of true variations in mass-loss rates at constant $\mathrm{M}_{\text {initial }}$ ? Do differences in composition or environment between these clusters affect mass-loss rates by up to $10 \%$ ? Or is mass loss stochastic in nature?

To begin to address both of these limitations, Cummings et al. (2015; 2016a; 2016b) have searched for new massive white dwarfs and have done detailed self-consistent analysis of both the white dwarf spectroscopy and open cluster photometry. For the wellstudied brightest white dwarfs, however, the parameters from the identical spectroscopic analysis in Gianninas et al. (2011) were used. Cummings et al. (2016a) focused in particular on self-consistently analyzing all publicly available raw spectroscopy of intermediate $\left(0.7\right.$ to $\left.1.0 \mathrm{M}_{\odot}\right)$ and high-mass $\left(>1.0 \mathrm{M}_{\odot}\right)$ white dwarfs using the white dwarf atmospheric models of Tremblay et al. (2011) with the Stark profiles of Tremblay \& Bergeron 
(2009) and the automated fitting techniques of Bergeron et al. (1992). The inferred progenitor masses are highly sensitive to systematic errors in the host cluster ages. Therefore, Cummings et al. (2016a) used multi-color UBV analysis, with detailed consideration of peculiar stars, the color dependence of reddening (see Fernie 1963), and the metallicity when available, to do high-precision turnoff isochronal ages of a wide number of star clusters. To consider the effects of the adopted evolutionary models, isochrones from both the Yi et al. (2001, hereafter $\mathrm{Y}^{2}$ ) models and the PARSEC models from Bressan et al. (2012) were used.

Figure 1 compares the IFMR from Cummings et al. (2015; 2016a; 2016b) and newer data from M7 (Cummings et al. in prep.) for $\mathrm{Y}^{2}$ cluster ages to the IFMR of Salaris et al. (2009). While there is still potentially important scatter in the relation, it is $\sim 50 \%$ less than that previously observed and can now predominantly be explained by the remaining errors and cluster non-member contamination. This suggests that the source of scatter in previous IFMR was dominated by systematics and that stellar mass loss is not stochastic.

Figure 1 also shows that progress has been made with finding white dwarfs in star clusters at masses greater than that of the long known LB 1497 in the Pleiades (>1.046 $\mathrm{M}_{\odot}$ ). These include several from 1.05 to $1.1 \mathrm{M}_{\odot}$ in NGC 2323 (Cummings et al. 2016a), NGC 2168 (Williams et al. 2009), and NGC 2287 (Dobbie et al. 2012). Then most recently in NGC 3532 at $1.123 \mathrm{M}_{\odot}$ (Raddi et al. 2016) and in NGC 2099 at $1.28 \mathrm{M}_{\odot}(\mathrm{Cummings}$ et al. 2016b; in blue). However, the very large sensitivity of cooling rate on mass at these highest masses makes the cooling age errors too large in this ultramassive white dwarf from NGC 2099. To reliably apply ultramassive white dwarfs to the IFMR, they must be hot and young before cooling age uncertainties have time to grow with age (Althaus et al. 2007; private communication). The most massive white dwarfs that can be applied to the IFMR are PG 0136+251 $\left(1.20 \mathrm{M}_{\odot}\right)$ and GD50 $\left(1.26 \mathrm{M}_{\odot}\right)$ and are both shown in green. They are young but do not remain in the Pleiades; Dobbie et al. (2006b) have argued kinematically that they are coeval and connected to the Pleiades.

\subsection{Remaining Challenges}

While important progress has been made in our understanding of the IFMR, several key limitations still remain. First, the evolutionary timescales of lower-mass stars $\left(<4 \mathrm{M}_{\odot}\right)$ are characterized well enough that using comparable evolutionary models to both derive isochronal cluster ages and interpret the resulting evolutionary timescales gives consistent progenitor masses. Any variations in the models are mitigated by the progenitor mass's lower sensitivity to evolutionary lifetime. However, when it comes to higher-mass progenitors the inferred progenitor masses are increasingly sensitive to the evolutionary models adopted. To illustrate this, in Figure 2 we compare the IFMR from Figure 1, which derived cluster ages from the $\mathrm{Y}^{2}$ isochrones, to the IFMR derived from PARSEC isochronal ages. For the typically young clusters these higher-mass white dwarfs are observed in, the PARSEC isochrones derive younger ages and infer more massive progenitors for the most massive cluster white dwarfs.

Second, many of the clusters analyzed for the IFMR are lacking precision spectroscopic metallicities. For clusters without metallicity information, the isochronal studies typically assume solar $[\mathrm{Fe} / \mathrm{H}]$. Therefore, this overestimates the isochronal ages in metal-rich clusters and underestimates them in metal-poor clusters. This will artificially increase the observed scatter in the IFMR. Furthermore, metallicity itself plays an important role in both mass loss and core mass growth during stellar evolution. This leads to more metal-rich stars losing more mass throughout their evolution giving lower mass white dwarfs, introducing an intrinsic scatter in the IFMR (see Marigo et al. 2007; Meng et al. 2008; Doherty et al. 2015; Romero et al. 2015). Detection of this scatter's correlation with 


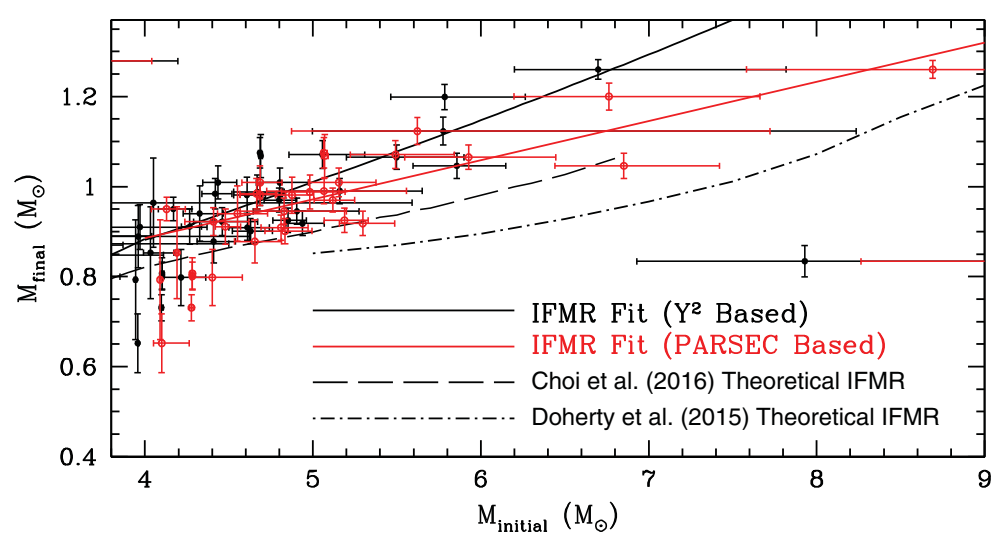

Figure 2. A look at the effects of adopting the $\mathrm{Y}^{2}$ and PARSEC stellar evolutionary models to fit the isochronal ages of the star clusters and infer progenitor masses. For clarity the focus is placed on the higher-mass stars where the effects are important, with the PARSEC models finding increasingly more massive progenitors. Comparisons to theoretical IFMR at solar metallicity are shown from Choi et al. (2016) and Doherty et al. (2015). Important systematic differences between the semi-empirical and theoretical IFMRs remain. (See online version for Figure 2 in color.)

cluster metallicity would provide critical constraints on our understanding of metallicity's effects on stellar evolution.

Lastly, the highest mass white dwarfs are still elusive. This is for a number of reasons. First, they are far rarer because there are very few stars with masses greater than 6 $\mathrm{M}_{\odot}$. Second, for compact objects like white dwarfs, the most massive ones also have the smallest radius, making them far less luminous than the most common intermediatemass white dwarfs. Third, observations of white dwarfs in the field have found that in addition to the high-mass ejected white dwarfs from the Pleiades (Dobbie et al. 2006b), there are six intermediate-mass white dwarfs that are kinematically connected to the Hyades (see Tremblay et al. 2012). Are white dwarfs prone to being ejected out of open clusters after formation? For example, asymmetric mass loss during their formation could provide strong enough velocity kicks to eject many white dwarfs soon after their formation (Fellhauer et al. 2003). To best overcome these issues and the very large cooling age errors in older ultramassive white dwarfs, the search for ultramassive white dwarfs should focus on young 50 to 100 Myr old clusters. These white dwarfs will be hotter and brighter, have minimal cooling age errors, and will not have had significant time to be ejected from their host cluster.

\section{Applications of the IFMR}

The most direct application of the IFMR is to measure total integrated mass loss across a star's lifetime and how it is sensitive to initial mass (and metallicity). Figure 3 looks at what percentage of a star's mass is lost throughout its lifetime versus $\mathrm{M}_{\text {initial }}$. This shows that from $\mathrm{M}_{\text {initial }}$ of 1.75 to $7 \mathrm{M}_{\odot}$ the total mass loss rapidly increases from $65 \%$ to over $80 \%$. Furthermore, to take into consideration the sensitivity to the adopted stellar model, there are two sets of data at the highest masses. The PARSEC models infer lower white dwarf masses for a given $\mathrm{M}_{\text {initial }}$, and hence a marginally higher mass loss. Because stellar mass loss occurs predominantly during the AGB phase, this provides critical information for understanding this complex stellar phase. Additionally, with PNe it gives a detailed 


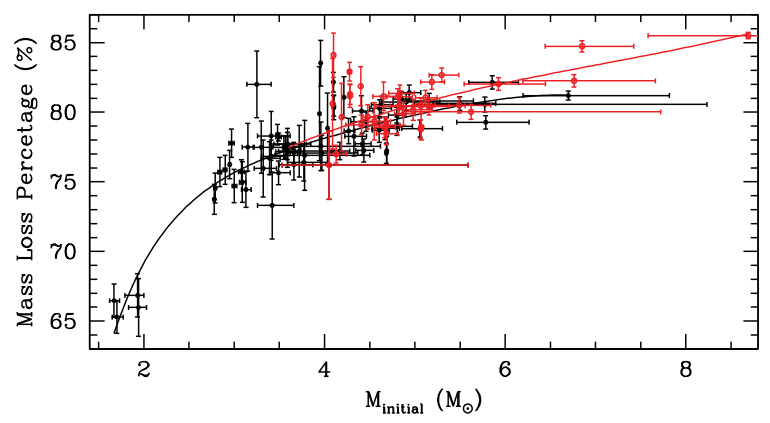

Figure 3. Comparison of the $\mathrm{M}_{\text {initial }}$ of a star to the total percentage of mass lost throughout its lifetime. As in Figure 2, the $\mathrm{Y}^{2}$ IFMR is in black and the PARSEC IFMR (shown for higher-mass stars only) is in open red, both with the mean relation shown in solid lines of corresponding color. (See online version for Figure 3 in color.)

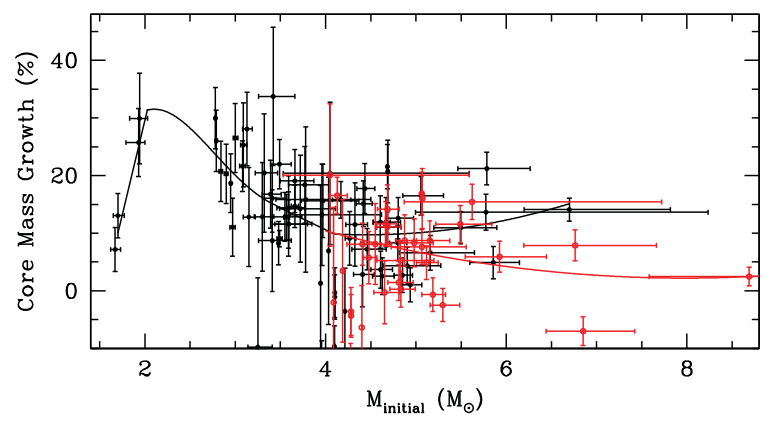

Figure 4. Comparison of $\mathrm{M}_{\mathrm{initial}}$ of a star to how much the core mass grows during the TP-AGB phase. As in Figures 2 and 3, the $\mathrm{Y}^{2}$ IFMR is in black and the PARSEC IFMR (shown for higher-mass stars only) is in open red, both with the mean relation shown in solid lines of corresponding color. (See online version for Figure 4 in color.)

measurement of the amount of mass that will surround (but not completely be ionized by) the hot central stars.

In the AGB a poorly constrained parameter is the amount of core mass growth that occurs during the thermally pulsing AGB (TP-AGB) phase. Modern stellar evolutionary models have reassuring agreement on the core masses at the first thermal pulse of the AGB $\left(\mathrm{M}_{1 \mathrm{stTP}}\right)$. However, note that variations that are seen in published models are more the result of differences in how different groups define this point in evolution and what layers are included in the core, rather than resulting differences in the predictions of stellar evolution (e.g., Marigo et al. 2013 versus Choi et al. 2016).

Figure 4 looks at this resulting core mass growth, in terms of $\left(\mathrm{M}_{\text {final }}-\mathrm{M}_{1 \mathrm{stTP}}\right) / \mathrm{M}_{1 \mathrm{stTP}}$. Again, the IFMR from both evolutionary models are considered, and while this resulted in important differences in the previous diagrams, here the differences are more telling. The $\mathrm{Y}^{2}$ models predict that the core masses grow significantly in the higher-mass stars, while the PARSEC models predict that the core masses do not grow and at the highest masses may decrease in mass during the TP-AGB. What is telling about this is that for the highest-mass AGB stars the thermally pulsing phase is very rapid. The phase is rapid enough that, unlike in lower mass stars, there is little to no time for the core masses to grow (e.g., Doherty et al. 2015). Any theoretical uncertainties remaining are likely not large enough to produce $\geqslant 10 \%$ core mass growth at $\mathrm{M}_{\text {initial }}>6 \mathrm{M}_{\odot}$. This strongly 
suggests that the $\mathrm{Y}^{2}$ evolutionary models are underestimating the progenitor masses for the highest mass white dwarfs, while the PARSEC models are more appropriate.

\section{Conclusions}

The IFMR is valuable for our understanding of stellar evolution. It was a longstanding challenge to find and spectroscopically observe a large population of white dwarfs within star clusters. With the advent of larger telescopes, wide-field imaging and multi-object spectrographs, the white dwarf sample greatly increased but significant scatter still remained in the relation. Within the past 5 years the sample size in the IFMR has continued to increase, and with the more advanced models and analysis techniques uniformly applied to both the spectroscopy and photometry, there has been a great reduction in the scatter.

While the highest-mass white dwarfs still remain elusive, addressing the remaining challenges of the IFMR will be more successful with the greatly diminished effects of systematics. These challenges include the remaining systematics between models used to infer progenitor masses, and the effects of metallicity for precise age dating and to test for metallicity's effect on the IFMR.

The diminished scatter of the current IFMR also makes the application of the relation more instructive. The applications of the IFMR to mass-loss rates across a broad range of $\mathrm{M}_{\text {initial }}$ shows that from $\mathrm{M}_{\text {initial }}$ of $1.75 \mathrm{M}_{\odot}$ to $7 \mathrm{M}_{\odot}$ the total mass loss percentage increased from $65 \%$ to greater than $80 \%$. Comparison of the IFMR to the $\mathrm{M}_{1 \mathrm{stTP}}$ also helps to illustrate the growth of core mass during the complex TP-AGB phase.

\section{References}

Althaus, L. G., García-Berro, E., Isern, J., Córsico, A., \& Rohrmann, R. 2007, A\&A, 465, 249

Beauchamp, A., Wesemael, F., Bergeron, P., Liebert, J., \& Saffer, R. A. 1996, Hydrogen Deficient Stars, 96, 295

Bergeron, P., Saffer, R. A., \& Liebert, J. 1992, ApJ, 394, 228

Bergeron, P., Liebert, J., \& Fulbright, M. S. 1995, ApJ, 444, 810

Bressan, A., Marigo, P., Girardi, L., et al. 2012, MNRAS, 427, 127

Casewell, S. L., Dobbie, P. D., Napiwotzki, R., et al. 2009, MNRAS, 395, 1795

Catalán, S., Isern, J., García-Berro, E., \& Ribas, I. 2008, MNRAS, 387, 1693

Choi, J., Dotter, A., Conroy, C., et al. 2016, ApJ, 823, 102

Ciardullo, R. 2010, PASA, 27, 149

Cummings, J. D., Kalirai, J. S., Tremblay, P.-E., \& Ramirez-Ruiz, E. 2015, ApJ, 807, 90

Cummings, J. D., Kalirai, J. S., Tremblay, P.-E., \& Ramirez-Ruiz, E. 2016a, ApJ, 818, 84

Cummings, J. D., et al. 2016b, ApJ, 820, L18

Dobbie, P. D., Napiwotzki, R., Burleigh, M. R., et al. 2006a, MNRAS, 369, 383

Dobbie, P. D., Napiwotzki, R., Lodieu, N., et al. 2006b, MNRAS, 373, L45

Dobbie, P. D., Day-Jones, A., Williams, K. A., et al. 2012, MNRAS, 423, 2815

Doherty, C., Gil-Pons, P., Siess, L., Lattanzio, J., \& Lau, H. 2015, MNRAS, 446, 2599

Eggen, O. J. \& Greenstein, J. L. 1965, ApJ, 141, 83

Fellhauer, M., Lin, D. N. C., Bolte, M., Aarseth, S. J., \& Williams, K. A. 2003, ApJ, 595, L53

Fernie, J. D. 1963, AJ, 68, 780

Fontaine, G., Brassard, P., \& Bergeron, P. 2001, PASP, 113, 409

Fusi-Pecci, F. \& Renzini, A. 1976, A\&A, 46, 447

Garcia-Berro, E., Isern, J., \& Hernanz, M. 1997, MNRAS, 289, 973

Gianninas, A., Bergeron, P., \& Ruiz, M. T. 2011, ApJ, 743, 138

Greggio, L. 2010, MNRAS, 406, 22

Kalirai, J. S., Richer, H. B., Reitzel, D., et al. 2005, ApJ, 618, L123

Kalirai, J. S., Bergeron, P., Hansen, B. M. S., et al. 2007, ApJ, 671, 748 
Kalirai, J. S., Hansen, B. M. S., Kelson, D. D., et al. 2008, ApJ, 676, 594

Kalirai, J. S., Saul Davis, D., Richer, H. B., et al. 2009, ApJ, 705, 408

Kalirai, J. S., Marigo, P., \& Tremblay, P.-E. 2014, ApJ, 782, 17

Koester, D. \& Reimers, D. 1993, A\&A, 275, 479

Koester, D. \& Reimers, D. 1996, A\&A, 313, 810

Marigo, P. \& Girardi, L. 2007, A\&A, 469, 239

Marigo, P., Bressan, A., Nanni, A., Girardi, L., \& Pumo, M. L. 2013, MNRAS, 434, 488

Meng, X., Chen, X., \& Han, Z. 2008, A\&A, 487, 625

Paczyński, B. 1970, AcA, 20, 47

Raddi, R., Catalán, S., Gänsicke, B. T., et al. 2016, MNRAS, 457, 1988

Reid, I. N. 1996, AJ, 111, 2000

Reimers, D. \& Koester, D. 1989, A\&A, 218, 118

Romero, A. D., Campos, F., \& Kepler, S. O. 2015, MNRAS, 450, 3708

Salaris, M., Serenelli, A., Weiss, A., \& Miller Bertolami, M. 2009, ApJ, 692, 1013

Schulz, H. 1976, Paper presented at Second Europ. Workshop on White Dwarfs, Rome

Sweeney, M. A. 1976, A\&A, 49, 375

Tremblay, P.-E. \& Bergeron, P. 2009, ApJ, 696, 1755

Tremblay, P.-E., Bergeron, P., \& Gianninas, A. 2011, ApJ, 730, 128

Tremblay, P.-E., Schilbach, E., Röser, S., et al. 2012, A\&A, 547, A99

Tremblay, P.-E., Kalirai, J., Soderblom, D., Cignoni, M., \& Cummings, J. 2014, ApJ, 791, 92

Tremblay, P.-E., Cummings, J., Kalirai, J. S., et al. 2016, MNRAS, 461, 2100

van Altena, W. F. 1969, AJ, 74, 2

Wegner, G. 1983, AJ, 88, 109

Wegner, G., Reid, I. N., \& McMahan, R. K. 1989, LNP, 328, 378

Weidemann, V. 1977, A\&A, 59, 411

Weidemann, V. 2000, A\&A, 363, 647

Williams, K. A., Bolte, M., \& Koester, D. 2004, ApJ, 615, L49

Williams, K. A., Bolte, M., \& Koester, D. 2009, ApJ, 693, 355

Wood, P. R. \& Cahn, J. H. 1977, ApJ, 211, 499

Yi, S., Demarque, P., Kim, Y.-C., et al. 2001, ApJS, 136, 417

\section{Discussion}

MUTHUMARIAPPAN: How do the binary interaction and common envelope evolution influence the initial and final mass relation?

Q: I am surprised to see that your plots start at $1.5-1.75 \mathrm{M}_{\odot}$ in initial mass. If you consider the peak mass of $0.6 \mathrm{M}_{\odot}$ observed in WDs, it seems that most PNe should come from $2 \mathrm{M}_{\odot}$ progenitor stars. Is this the case?

Cummings: The mean mass is roughly $0.6 \mathrm{M}_{\odot}$, but the peak (most common) massis roughly 0.56 to $0.58 \mathrm{M}_{\odot}$. This gives progenitors of $\sim 1.5 \mathrm{M}_{\odot}$ as likely the most common $\mathrm{PN}$ progenitors.

Mendez: What is the final mass for an initial mass of $1 \mathrm{M}_{\odot}$ and what would be the error bar?

Cummings: White dwarfs with progenitors of this mass have been observed in M4. While its very low metallicity may affect our comparison of this to a solar metallicity star, but it suggests that they will for 0.55 to $0.50 \mathrm{M}_{\odot}$ white dwarfs. See Kalirai et al. (2009).

Q: What is the metallicity range you are sampling with these clusters and can the massloss rate dependency on metallicity explain the scatter or some of the scatter you are seeing in the IFMR? 